\title{
CONDITIONS FOR CONVERGENCE OF THE FUNDAMENTAL MATRIX OF LINEAR TIME-INVARIANT TIME-DELAYED SINGULAR SYSTEMS
}

\author{
BRANKO SARIC
}

Received 31 January 2001 and in revised form 13 December 2001

\begin{abstract}
In the introductory part of this paper the so-called general Bromwich-Wagner's theorem has been generalized. Throughout the main part of the paper, an attempt has been made to derive conditions for convergence of the fundamental matrix of linear time-invariant timedelayed singular systems. The paper ends with a counterexample pointing out the fact that certain results of the theory of linear time-invariant singular systems are incorrect.
\end{abstract}

2000 Mathematics Subject Classification: 93C05, 34A30, 34D05.

1. Introduction. The introductory part is provided to give the generalization of the so-called general Bromwich-Wagner's theorem from Cauchy's calculus of residues, which has proven to be a powerful tool in the Laplace transforms theory. The main part is concerned with the theory of singular systems. On the basis of the result worded in the form of the theorem at the very beginning, throughout the main part of the paper, an attempt has been made to derive conditions for convergence of the fundamental matrix of linear time-invariant time-delayed singular systems. The paper ends with a counterexample pointing out the fact that certain results of the theory of linear timeinvariant singular systems, as for example a condition for the existence of infinite frequency dynamic modes (of impulsive modes) in a solution formulation of linear time-invariant singular systems, are incorrect.

In the complex functions theory, more precisely in the theory of Cauchy's calculus of residues, the following result, which is well known as the general Bromwich-Wagner's theorem (see [4, Theorem 1, page 215]), is a fundamental result from the viewpoint of determining the function $t \mapsto f(t)$ whose Laplace transform is $z \mapsto F(z)$.

THEOREM 1.1. Let $F$ be a function with the following properties:

(1) $F$ is an analytic function on the domain $G=\{z \mid \operatorname{Re} z<c\}$ inside which it has finitely many singularities $z_{1}, z_{2}, \ldots, z_{n}$;

(2) on the straight line $\operatorname{Re} z=c, F$ has finitely many simple poles $a_{1}, a_{2}, \ldots, a_{m}$;

(3) $\lim _{|z| \rightarrow+\infty} F(z)=0$ in the half-plane $G$.

If $\alpha>1$, then the following equality holds:

$$
\operatorname{vp} \int_{c-i \infty}^{c+i \infty} \alpha^{z} F(z) d z=2 \pi i \sum_{k=1}^{n} \operatorname{Res}_{z=z_{k}}\left[\alpha^{z} F(z)\right]+\pi i \sum_{k=1}^{m} \operatorname{Res}_{z=a_{k}}\left[\alpha^{z} F(z)\right],
$$

where $\operatorname{vp} \int_{\mathcal{c}-i \infty}^{c+i \infty} \alpha^{z} F(z) d z$ is the so-called Couchy's principal value of the improper integral $\int_{\mathcal{C}-i \infty}^{c+i \infty} \alpha^{z} F(z) d z$. 
The more general case in which a function $z \mapsto F(z)$ has an infinite but countable set of singularities is also applicable [1]. Thus, it is of a general interest to prove the theorem that is slightly more general with respect to Theorem 1.1. On the basis of this theorem, in what follows, we will derive the result which is more general with respect to that of [6, equation (5.17)] giving an exponential assessment for integral convergence of the fundamental matrix of a class of linear time-delayed regular systems in the proof of [6, Theorem 5.1, page 293] and which can be applied in stability analysis of motions (of solutions) of a class of linear time-delayed singular systems.

THEOREM 1.2. Let $F(z)$ be a function with the following properties:

(1) $F(z)$ is an analytic function on the domain $G=\{z \mid \operatorname{Re} z<c\}$ inside which it has an infinite but countable set of singularities $z_{1}, z_{2}, \ldots$;

(2) on the straight line $\operatorname{Re} z=c$ the function $F$ has an infinite but countable set of simple poles $a_{1}, a_{2}, \ldots$;

(3) $\lim _{|z| \rightarrow+\infty} F(z)=0$ in the half-plane $G$;

(4) the disposition of singularities of the function $F(z)$ on the complex plane is such that there exists a half-circular contour of integration $\Omega_{r}$,

$$
\Omega_{r}=\{z|| z-c \mid=r, \operatorname{Re} z \leq c\}
$$

by which the singularities of $F(z)$ are divided in such a way that no singularities lie onto $\Omega_{r}$, and in the domain bounded by $\Omega_{r}$ and the straight line $\operatorname{Re} z=c$, the singularities lying on $\operatorname{Re} z=c$ are isolated ones.

If $\alpha>1$, then the following equality holds:

$$
\operatorname{vp} \int_{c-i \infty}^{c+i \infty} \alpha^{z} F(z) d z=2 \pi i \sum_{k=1}^{+\infty} \operatorname{Res}_{z=z_{k}}\left[\alpha^{z} F(z)\right]+\pi i \sum_{k=1}^{+\infty} \operatorname{Res}_{z=a_{k}}\left[\alpha^{z} F(z)\right] .
$$

Proof. On the one hand, according to condition (4) of the theorem, the integral equality

$$
\int_{G_{r}}^{\circlearrowleft} \alpha^{z} F(z) d z=\sum_{k=0}^{m} \int_{l_{k}}^{\dagger} \alpha^{z} F(z) d z+\sum_{k=1}^{m} \int_{g_{k}}^{\curvearrowright} \alpha^{z} F(z) d z+\int_{\Omega_{r}}^{\curvearrowleft} \alpha^{z} F(z) d z,
$$

(the symbol $\int_{G_{r}}^{\cup}$ denotes an integration over a closed contour of integration $G_{r}$, in this case in the positive mathematical direction) where the contour of integration $G_{r}=\Omega_{r} \cup l_{0} \cup g_{1} \cup l_{1} \cup g_{2} \cup \cdots \cup l_{m}$ consists of half-circular contours of integration $\Omega_{r}=\{z|| z-c \mid=r, \operatorname{Re} z \leq c\} \quad\left(r \in \mathbb{R}_{+}^{1}\right)$ and $g_{k}=\left\{z|| z-a_{k} \mid=\delta\left(\delta \in \mathbb{R}_{+}^{1}\right), \operatorname{Re} z \leq c\right\}$ $(k=1,2, \ldots, m)$ having no common points, as well as of segments

$$
\begin{gathered}
l_{0}=\left\{z \mid \operatorname{Re} z=c, \operatorname{Im} z \in\left[-r, \operatorname{Im} a_{1}-\delta\right]\right\}, \\
l_{k}=\left\{z \mid \operatorname{Re} z=c, \operatorname{Im} z \in\left[\operatorname{Im} a_{k}+\delta, \operatorname{Im} a_{k+1}-\delta\right]\right\} \quad(k=1,2, \ldots, m-1), \\
l_{m}=\left\{z \mid \operatorname{Re} z=c, \operatorname{Im} z \in\left[\operatorname{Im} a_{m}+\delta, r\right]\right\},
\end{gathered}
$$


is reduced by one-to-one mapping $z-c=i$ s the complex plane $z$ onto the complex plane $s$ to the integral equality

$$
\begin{aligned}
e^{c \log \alpha} \int_{\Gamma_{r}}^{\cup} F(c+i s) e^{i s \log \alpha} d s= & e^{c \log \alpha} \sum_{k=0}^{m} \int_{\lambda_{k}}^{\rightarrow} F(c+i s) e^{i s \log \alpha} d s \\
& +e^{c \log \alpha} \sum_{k=1}^{m} \int_{\gamma_{k}}^{\curvearrowright} F(c+i s) e^{i s \log \alpha} d s \\
& +e^{c \log \alpha} \int_{\omega_{r}}^{\curvearrowleft} F(c+i s) e^{i s \log \alpha} d s,
\end{aligned}
$$

where the contour of integration $\Gamma_{r}=\omega_{r} \cup \lambda_{0} \cup \gamma_{1} \cup \lambda_{1} \cup \gamma_{2} \cup \cdots \cup \lambda_{m}$ consists of halfcircular contours of integration $\omega_{r}=\{s|| s \mid=r$, $\operatorname{Im} s \geq 0\}$ and $\gamma_{k}=\left\{s|| s-\operatorname{Im} a_{k} \mid=\right.$ $\delta, \operatorname{Im} s \geq 0\}(k=1,2, \ldots, m)$, as well as of segments

$$
\begin{gathered}
\lambda_{0}=\left\{s \mid s \in\left[-r, \operatorname{Im} a_{1}-\delta\right]\right\}, \\
\lambda_{k}=\left\{s \mid s \in\left[\operatorname{Im} a_{k}+\delta, \operatorname{Im} a_{k+1}-\delta\right]\right\} \quad(k=1,2, \ldots, m-1), \\
\lambda_{m}=\left\{s \mid s \in\left[\operatorname{Im} a_{m}+\delta, r\right]\right\} .
\end{gathered}
$$

On the other hand, from the first Jordan lemma (see [4, Theorem 1, page 52]) and condition (2) of the theorem, it follows that

$$
\lim _{\delta \rightarrow 0^{+}} \sum_{k=1}^{m} \int_{\gamma_{k}}^{\curvearrowright} F(c+i s) e^{i s \log \alpha} d s=-\pi i \sum_{k=1}^{m} \operatorname{Res}_{s=a_{k}}\left[F(c+i s) e^{i s \log \alpha}\right] .
$$

Also, from the third Jordan lemma (see [4, Theorem 3, page 52]) and condition (3) of the theorem it follows that

$$
\lim _{r \rightarrow+\infty} \int_{\omega_{r}}^{\curvearrowleft} F(c+i s) e^{i s \log \alpha} d s=0 .
$$

Finally, on the basis of the general Cauchy theorem (see [4, Theorem 1, page 43]), we obtain

$$
\begin{aligned}
\operatorname{vp} \int_{c-i \infty}^{c+i \infty} \alpha^{z} F(z) d z= & i e^{c \log \alpha} \operatorname{vp} \int_{-\infty}^{+\infty} F(c+i s) e^{i s \log \alpha} d s \\
= & 2 \pi i \sum_{k=1}^{+\infty} \operatorname{Res}_{s=s_{k}}\left[i e^{(c+i s) \log \alpha} F(c+i s)\right] \\
& +\pi i \sum_{k=1}^{+\infty} \operatorname{Res}_{s=a_{k}}\left[i e^{(c+i s) \log \alpha} F(c+i s)\right],
\end{aligned}
$$

where the sums of residues are calculated with respect to all singularities of the function $s \mapsto i e^{(c+i s) \log \alpha} F(c+i s)$ in the open upper half-plane as well as onto the real axis, respectively.

Since on the basis of a low of transformation

$$
s=-i(z-c)=\operatorname{Im} z+i(c-\operatorname{Re} z),
$$


all singularities of the function $\alpha^{z} F(z)$ in the half-plane $\operatorname{Re} z \leq c$ of the complex plane $z$ are at the same time singularities of the function $s \mapsto i e^{(c+i s) \log \alpha} F(c+i s)$ in the upper half-plane $\operatorname{Im} s \geq 0$ of the complex plane $s$, thus the equality previously derived is reduced to that of the theorem.

If $\alpha=e^{t}(t>0)$, then it follows from the proof of Theorem 1.2 that

$$
\lim _{r \rightarrow+\infty} \int_{\Omega_{r}}^{n} e^{z t} F(z) d z=0 .
$$

EXAMPLE 1.3. If $F(z)=1 / z$, then

$$
\begin{gathered}
\int_{c-i \infty}^{c+i \infty} \frac{e^{z t}}{z} d z= \begin{cases}2 \pi i & \text { for } c>0, \\
0 & \text { for } c<0,\end{cases} \\
\operatorname{vp} \int_{-i \infty}^{+i \infty} \frac{e^{z t}}{z} d z=i \pi .
\end{gathered}
$$

\section{The main results}

2.1. The fundamental matrix of linear time-invariant time-delayed singular systems. The fundamental matrix $\boldsymbol{\Phi}_{s}(t)$ of a linear time-invariant time-delayed system of differential-algebraic equations

$$
\begin{gathered}
\mathbf{P} \frac{d}{d t} \mathbf{x}_{0}(t)=\sum_{k=0}^{K} \mathbf{A}_{k} \mathbf{x}_{k}(t), \\
\mathbf{x}_{k}(t)=\mathbf{x}\left(t+\theta_{k}\right) \quad\left(\theta_{k} \in[-\mu, 0], 0=\left|\theta_{0}\right|<\left|\theta_{1}\right|<\cdots<\left|\theta_{K}\right|=\mu \in \mathbb{R}_{0+}^{1}\right), \\
\mathbf{x}_{0}\left(0^{+}\right)=\mathbf{x}_{\tilde{0}}, \quad \mathbf{x}_{0}(t)=\mathbf{x}_{\tilde{o}}(t) \quad \text { for } t \in(-\mu, 0),
\end{gathered}
$$

where $n \times n$ matrices $\mathbf{A}_{k}$ and a singular $n \times n$ matrix $\mathbf{P}$ are in the general case constant matrices defined on the field of the complex numbers, is a solution of the matrix differential equation

$$
\begin{gathered}
\frac{d}{d t} \boldsymbol{\Phi}_{s}(t) \mathbf{P}=\sum_{k=0}^{K} \boldsymbol{\Phi}_{s}\left(t+\theta_{k}\right) \mathbf{A}_{k}, \\
\left.\boldsymbol{\Phi}_{S}(t) \mathbf{P}\right|_{t=0^{+}}=\boldsymbol{\Phi}_{s}\left(0^{+}\right) \mathbf{P}, \quad \boldsymbol{\Phi}_{s}(t) \equiv \mathbf{0} \text { for } t<0,
\end{gathered}
$$

whenever it exists.

Namely, in the first step multiply both sides of system (2.1) on the left by matrix $\boldsymbol{\Phi}_{s}(t-s)$ and after that integrate it with respect to $s$ from 0 to $t$. In such a way, we obtain

$$
\lim _{\substack{\omega \rightarrow t^{-} \\ \omega_{0} \rightarrow 0^{+}}} \int_{\omega_{0}}^{\omega} \boldsymbol{\Phi}_{s}(t-s) \mathbf{P} \frac{d}{d s} \mathbf{x}_{0}(s) d s=\lim _{\substack{\omega \rightarrow t^{-} \\ \omega_{0} \rightarrow 0^{+}}} \int_{\omega_{0}}^{\omega} \sum_{k=0}^{K} \boldsymbol{\Phi}_{s}(t-s) \mathbf{A}_{k} \mathbf{x}_{k}(s) d s,
$$

that is,

$$
\boldsymbol{\Phi}_{s}\left(0^{+}\right) \mathbf{P} \mathbf{x}_{0}(t)=\boldsymbol{\Phi}_{s}(t) \mathbf{P} \mathbf{x}_{0}\left(0^{+}\right)+\sum_{k=0}^{K} \int_{\theta_{k}}^{0} \boldsymbol{\Phi}_{s}\left(t+\theta_{k}-s\right) \mathbf{A}_{k} \mathbf{x}_{\tilde{o}}(s) d s .
$$


According to the Laplace transform of the fundamental matrix $\boldsymbol{\Phi}_{S}(t)$

$$
\tilde{\boldsymbol{\Phi}}_{s}(z)=\boldsymbol{\Phi}_{s}\left(0^{+}\right) \mathbf{P}\left(z \mathbf{P}-\sum_{k=0}^{K} \mathbf{A}_{k} e^{z \theta_{k}}\right)^{-1},
$$

obtained by formal application of the Laplace transform in the course of solving the matrix differential equation (2.2), the characteristic equation of the system is

$$
\operatorname{det}\left(z \mathbf{P}-\sum_{k=0}^{K} \mathbf{A}_{k} e^{z \theta_{k}}\right)=0 .
$$

Note 2.1. It should be emphasized that for solving the matrix differential equation (2.2) by formal application of the Laplace transform, it is necessary to make an additional check of an integral convergence $\int_{0}^{+\infty} \boldsymbol{\Phi}_{s}(t) e^{-z t} d t$ in order for formality of the procedure to be justified. By formal application of the Laplace transform, we can directly come to solution (2.2) of system (2.1), too. Namely, for continuous solutions $\mathbf{x}_{0}(t)$ of system (2.1), and under the assumption that vector valued functions of initial states $\mathbf{x}_{\tilde{o}}(t) \in \bar{T}_{0-\mu} \rightarrow C^{n}$ of the system are elements of the functional space of functions $\tilde{\mathbf{x}}(\theta)$ which are continuous in the segment $[-\mu, 0]$ with the standard norm $\|\tilde{\mathbf{X}}(\theta)\|=\sup |\tilde{\mathbf{X}}(\theta)|$ (where $\theta \in[-\mu, 0]$ ), it can be shown by the method of partial integration that

$$
\begin{gathered}
\lim _{\substack{\omega \rightarrow+\infty \\
\omega \\
\omega_{0} \rightarrow 0^{+}}} \int_{\omega_{0}}^{\omega} \dot{\mathbf{x}}_{0}(t) e^{-z t} d t=-\mathbf{x}_{0}\left(0^{+}\right)+z \lim _{\substack{\omega \rightarrow+\infty \\
\omega \\
\omega_{0} \rightarrow 0^{+}}} \int_{\omega_{0}}^{\omega} \mathbf{x}_{0}(t) e^{-z t} d t, \\
\lim _{\substack{\omega \rightarrow+\infty \\
\omega_{0} \rightarrow 0^{+}}} \int_{\omega_{0}}^{\omega} \mathbf{x}_{k}(t) e^{-z t} d t=e^{z \theta_{k}}\left[\lim _{\substack{\omega \rightarrow+\infty \\
\omega_{0} \rightarrow 0^{+}}} \int_{\omega_{0}}^{\omega} \mathbf{x}_{0}(t) e^{-z t} d t+\int_{\theta_{k}}^{0} \mathbf{x}_{\tilde{o}}(t) e^{-z t} d t\right] .
\end{gathered}
$$

Thus

$$
\overline{\boldsymbol{\Phi}}_{s}(z) \lim _{\substack{\omega \rightarrow+\infty \\ \omega_{0} \rightarrow 0^{+}}} \int_{\omega_{0}}^{\omega} \mathbf{x}_{0}(t) e^{-z t} d t=\mathbf{p}(z),
$$

where $\overline{\boldsymbol{\Phi}}_{s}(z)=z \mathbf{P}-\sum_{k=0}^{K} \mathbf{A}_{k} e^{z \theta_{k}}$ and $\mathbf{p}(z)=\mathbf{P x}_{\tilde{0}}+\sum_{k=0}^{K} \mathbf{A}_{k} e^{z \theta_{k}} \int_{\theta_{k}}^{0} \mathbf{x}_{\tilde{o}}(t) e^{-z t} d t$.

On the basis of the inversion theorem [1, Theorem 1.1, page 18] for sufficiently large real constant $c \in \mathbb{R}_{+}^{1}$,

$$
\begin{aligned}
\boldsymbol{\Phi}_{s}\left(0^{+}\right) \mathbf{P} \mathbf{x}_{0}(t) & =\frac{1}{2 \pi i} \lim _{\omega \rightarrow+\infty} \int_{c-i \omega}^{c+i \omega} \boldsymbol{\Phi}_{s}\left(0^{+}\right) \mathbf{P} \overline{\mathbf{\Phi}}_{s}^{-1}(z) \mathbf{p}(z) e^{z t} d z \\
& =\frac{1}{2 \pi i} \lim _{\omega \rightarrow+\infty} \int_{c-i \omega}^{c+i \omega} \tilde{\boldsymbol{\Phi}}_{s}(z) \mathbf{p}(z) e^{z t} d z .
\end{aligned}
$$

According to the convolution theorem [1, Theorem 1.4, page 26], the right-hand side of the preceding relation is equivalent to the right-hand side of relation (2.4).

For a class of linear singular systems without delay $\mathbf{P} \dot{\mathbf{x}}(t)=\mathbf{A} \mathbf{x}(t)$, the fundamental matrix of the system $\boldsymbol{\Phi}_{s}(t)=\hat{\mathbf{P}}^{D} e^{\hat{\mathbf{P}}^{D} \hat{\mathbf{A}} t}$ for $t \geq 0$, where the matrix $\hat{\mathbf{P}}^{D}$ is a Drazin inverse matrix of $\hat{\mathbf{P}}, \hat{\mathbf{P}}=\mathbf{P}(\nu \mathbf{P}+\mathbf{A})^{-1}$, (of course if there exists a constant $v$ such that the matrix 
$(\nu \mathbf{P}+\mathbf{A})$ is an invertible one) and $\hat{\mathbf{A}}=\mathbf{A}(\nu \mathbf{P}+\mathbf{A})^{-1}$ is a matrix solution of the matrix differential equation $\dot{\boldsymbol{\Phi}}_{s}(t) \mathbf{P}=\boldsymbol{\Phi}_{s}(t) \mathbf{A}$ with the initial conditions $\boldsymbol{\Phi}_{s}\left(0^{+}\right) \mathbf{P}=\hat{\mathbf{P}}^{D} \mathbf{P}$ and $\boldsymbol{\Phi}_{s}(t) \equiv \mathbf{0}$ for $t<0$.

EXAMPLE 2.2. By a table of Laplace transforms of elementary functions, a fundamental matrix of the system $\mathbf{P} \dot{\mathbf{x}}(t)=\mathbf{A} \mathbf{x}(t)$, where $\mathbf{P}=\left[\begin{array}{ll}1 & 0 \\ 0 & 0\end{array}\right]$ and $\mathbf{A}=\left[\begin{array}{cc}-1 & 0 \\ 0 & 1\end{array}\right]$ as well as $\mathbf{P}(z \mathbf{P}-\mathbf{A})^{-1}=\left[\begin{array}{ll}1 & 0 \\ 0 & 0\end{array}\right]\left[\begin{array}{cc}1 /(z+1) & 0 \\ 0 & -1\end{array}\right]$, is of the matrix form $\boldsymbol{\Phi}_{s}(t)=\left[\begin{array}{cc}e^{-t} & 0 \\ 0 & 0\end{array}\right]$. In addition, it is also a solution of the differential matrix equation $\dot{\boldsymbol{\Phi}}_{s}(t) \mathbf{P}=\boldsymbol{\Phi}_{s}(t) \mathbf{A}$ with the initial condition $\boldsymbol{\Phi}_{s}\left(0^{+}\right) \mathbf{P}=\mathbf{P}$, since $\hat{\mathbf{P}}^{D} \mathbf{P}=\mathbf{P}$.

2.2. The conditions for convergence of the fundamental matrix of linear timeinvariant time-delayed singular systems. The matrix $\boldsymbol{\Phi}_{s}\left(0^{+}\right)$of the initial condition $\left.\boldsymbol{\Phi}_{s}(t) \mathbf{P}\right|_{t=0^{+}}=\boldsymbol{\Phi}_{s}\left(0^{+}\right) \mathbf{P}$ cannot be an arbitrarily chosen matrix but it has to belong to a set of matrices for which there exists a solution of the matrix differential equation (2.2). In that emphasized case, the Laplace transform $\tilde{\boldsymbol{\Phi}}_{s}(z)$ of the fundamental $n \times n$ matrix $\boldsymbol{\Phi}_{s}(t)$ of system (2.1) can be expressed in the form which is suitable for contour integration, that is, in the form

$$
\tilde{\boldsymbol{\Phi}}_{s}(z)=\frac{\boldsymbol{\Phi}_{s}\left(0^{+}\right)}{z}\left[\mathbf{E}+\sum_{k=0}^{K} \mathbf{A}_{k} e^{z \theta_{k}}\left(z \mathbf{P}-\sum_{k=0}^{K} \mathbf{A}_{k} e^{z \theta_{k}}\right)^{-1}\right],
$$

where $\mathbf{E}$ is the identity $n \times n$ matrix, from which it follows immediately that $\lim _{|z| \rightarrow+\infty} \tilde{\boldsymbol{\Phi}}_{s}(z)=0$ in the half-plain $\operatorname{Re} z \leq c$.

Namely, if

$$
\overline{\mathbf{G}}_{s}(z)=e^{z \mu} \overline{\mathbf{\Phi}}_{s}(z)=z e^{z \mu} \mathbf{P}-\sum_{k=0}^{K} \mathbf{A}_{k} e^{z \beta_{K-k}}=z e^{z \mu} \mathbf{P}-\sum_{k=0}^{K} \mathbf{A}_{K-k} e^{z \beta_{k}},
$$

where $\beta_{k}=\mu+\theta_{K-k}$ and $0=\beta_{0}<\beta_{1}<\cdots<\beta_{K}=\mu$, it follows that $\operatorname{det} \overline{\mathbf{G}}_{s}(z)=$ $e^{n z \mu} \operatorname{det} \overline{\boldsymbol{\Phi}}_{s}(z)$ and $\overline{\mathbf{G}}_{s}^{-1}(z)=e^{-z \mu} \overline{\boldsymbol{\Phi}}_{s}^{-1}(z)$, that is,

$$
\tilde{\boldsymbol{\Phi}}_{s}(z)=\frac{\boldsymbol{\Phi}_{s}\left(0^{+}\right)}{z}\left[\mathbf{E}+\sum_{k=0}^{K} \mathbf{A}_{K-k} e^{z \beta_{k}} \overline{\mathbf{G}}_{s}^{-1}(z)\right] .
$$

On the basis of the fact that exponential multipliers $e^{z \beta_{k}}$ in the half-plane $\operatorname{Re} z \leq c$ are bounded and the degrees of polynomials of minors of the matrix $\overline{\mathbf{G}}_{s}(z)$ are no higher than the degrees of polynomials of the polynomial function $\operatorname{det} \overline{\mathbf{G}}_{s}(z)$, it follows explicitly that in the half-plane $\operatorname{Re} z \leq c$

$$
\lim _{|z| \rightarrow+\infty} \tilde{\boldsymbol{\Phi}}_{S}(z)=\lim _{|z| \rightarrow+\infty}\left\{\frac{\boldsymbol{\Phi}_{S}\left(0^{+}\right)}{z}\left[\mathbf{E}+\sum_{k=0}^{K} \mathbf{A}_{K-k} e^{z \beta_{k}} \overline{\mathbf{G}}_{s}^{-1}(z)\right]\right\}=\mathbf{0} .
$$

In view of the fact that for each $k$ and $\theta_{k} \leq t \leq 0$, the exponential multipliers $e^{z\left(\mu+\theta_{k}-t\right)}$ are also bounded in the half-plane $\operatorname{Re} z \leq c$, it follows immediately that the functional expression $\lim _{|z| \rightarrow+\infty} \overline{\mathbf{p}}(z)=\lim _{|z| \rightarrow+\infty} \mathbf{p}(z) e^{z \mu}$ converges in the half-plane $\operatorname{Re} z \leq c$. 
Since, on the basis of [1, Theorem 12.13, pages 457-458], there exists a half-circular contour of integration $\Omega_{r}$ by which the singularities of $\tilde{\boldsymbol{\Phi}}_{s}(z)$ are divided in the halfplane $\operatorname{Re} z \leq c$ in such a way that no singularities lie on the contour $\Omega_{r}$, it follows from the result of Theorem 1.2 that if $\tilde{\boldsymbol{\Phi}}_{s}(z)$ is a regular matrix function in the half-plane $\operatorname{Re} z \geq c\left(c \in \mathbb{R}_{+}^{1}\right)$, more precisely if the characteristic quasi-polynomial of $\tilde{\boldsymbol{\Phi}}_{s}(z)$ has a principal term (see [1, Theorem 13.1, pages 480-481]), then for $t>\mu$

$$
\begin{aligned}
\boldsymbol{\Phi}_{S}\left(0^{+}\right) \mathbf{P} \mathbf{x}_{0}(t)= & \frac{1}{2 \pi i} \int_{c^{\prime}-i \infty}^{c^{\prime}+i \infty} \tilde{\boldsymbol{\Phi}}_{S}(z) \overline{\mathbf{p}}(z) e^{z(t-\mu)} d z \\
& +\sum_{n=1}^{N} \operatorname{Res}_{\substack{z=z_{n} \\
\left(\operatorname{Re} z_{n}>c^{\prime}\right)}}\left[\tilde{\boldsymbol{\Phi}}_{S}(z) \overline{\mathbf{p}}(z) e^{z(t-\mu)}\right],
\end{aligned}
$$

where $c^{\prime}<c$. As $\mathbf{p}(z)=\mathbf{P} \mathbf{x}_{0}\left(0^{+}\right)+\sum_{k=0}^{K} \mathbf{A}_{k} e^{z \theta_{k}} \int_{\theta_{k}}^{0} \mathbf{x}_{\tilde{o}}(t) e^{-z t} d t$ and

$$
\left|\int_{\theta_{k}}^{0} \mathbf{x}_{\tilde{o}}(t) e^{-z\left(t-\theta_{k}\right)} d t\right| \leq\left\|\mathbf{x}_{\tilde{0}}\right\|\left|\int_{\theta_{k}}^{0} e^{-z\left(t-\theta_{k}\right)} d t\right|=|| \mathbf{x}_{\tilde{0}} \|\left|\frac{1}{z}\left(1-e^{-z \theta_{k}}\right)\right|,
$$

as well as

$$
\left|\frac{1}{2 \pi i} \int_{c^{\prime}-i \infty}^{c^{\prime}+i \infty} \tilde{\boldsymbol{\Phi}}_{s}(z) \mathbf{p}(z) e^{z t} d z\right| \leq a\left\|\mathbf{x}_{0}\right\| e^{c^{\prime} t}
$$

where $a=(1 / 2 \pi i) \int_{\mathcal{c}^{\prime}-i \infty}^{c^{\prime}+i \infty}\left|\tilde{\boldsymbol{\Phi}}_{S}(z)\right|\left(|\mathbf{P}|+\sum_{k=0}^{K}\left|\mathbf{A}_{k}\right|\left|z^{-1}\left(1-e^{-z \theta_{k}}\right)\right|\right) d z$, then it follows finally that for $t>\mu$

$$
\left|\boldsymbol{\Phi}_{s}\left(0^{+}\right) \mathbf{P} \mathbf{x}_{0}(t)-\sum_{n=1}^{N} \operatorname{Res}_{\substack{z=z_{n} \\\left(\operatorname{Re} z_{n}>c^{\prime}\right)}}\left[\tilde{\boldsymbol{\Phi}}_{s}(z) \mathbf{p}(z) e^{z t}\right]\right| \leq a\left\|\mathbf{x}_{0}\right\| e^{c^{\prime} t}
$$

Note that Theorem 1.2 can be similarly applied to the integral equality, instead to equality (2.9), can be applied to the integral equality too

$$
\boldsymbol{\Phi}_{s}\left(0^{+}\right) \mathbf{P} \int_{0}^{t} \mathbf{x}_{0}(\tau) d \tau=\frac{1}{2 \pi i} \lim _{\omega \rightarrow+\infty} \int_{c-i \omega}^{c+i \omega} \frac{\tilde{\mathbf{\Phi}}_{s}(z)}{z} \mathbf{p}(z) e^{z t} d z
$$

In that emphasized case, for $t>\mu$, it holds that

$$
\left|\boldsymbol{\Phi}_{s}\left(0^{+}\right) \mathbf{P} \int_{0}^{t} \mathbf{x}_{0}(\boldsymbol{\tau}) d \boldsymbol{\tau}-\sum_{n=1}^{N} \underset{\substack{\left.z=z_{n} \\ \operatorname{Re} z_{n}>c^{\prime}\right)}}{\operatorname{Res}}\left[\frac{\tilde{\boldsymbol{\Phi}}_{s}(z)}{z} \mathbf{p}(z) e^{z t}\right]\right| \leq \hat{a}\left\|\mathbf{x}_{0}\right\| e^{c^{\prime} t},
$$

where $\hat{a}=(1 / 2 \pi i) \int_{c^{\prime}-i \infty}^{c^{\prime}+i \infty}\left(\left|\tilde{\mathbf{\Phi}}_{s}(z)\right| /|z|\right)\left(|\mathbf{P}|+\sum_{k=0}^{K}\left|\mathbf{A}_{k}\right|\left|z^{-1}\left(1-e^{-z \theta_{k}}\right)\right|\right) d z$. 
On the basis of the results (2.17) and (2.19), it follows that if $\operatorname{Re} z_{n}<c^{\prime}<0$, where $z_{n}$ are zeros of the characteristic quasi-polynomial of system (2.1), then

$$
\lim _{t \rightarrow+\infty} \boldsymbol{\Phi}_{s}\left(0^{+}\right) \mathbf{P} \mathbf{x}_{0}(t)=\mathbf{0}, \quad \lim _{t \rightarrow+\infty} \boldsymbol{\Phi}_{s}\left(0^{+}\right) \mathbf{P} \int_{0}^{t} \mathbf{x}_{0}(\boldsymbol{\tau}) d \boldsymbol{\tau}=\tilde{\boldsymbol{\Phi}}_{s}\left(0^{+}\right) \mathbf{p}(0),
$$

respectively.

The preceding results can be generalized in a sense of obtaining the general conditions for convergence of the fundamental matrix $\boldsymbol{\Phi}_{s}(t)$ of system (2.1). Namely, on the one hand, as a result of successive differentiation of both sides of the well-known formula for the Laplace transform of the $n$th derivative of an arbitrary function $t \mapsto f(t)$

$$
\int_{0}^{+\infty} \frac{d^{n}}{(d \tau)^{n}} f(\tau) e^{-z \tau} d \tau=z^{n} \tilde{f}(z)-\sum_{k=0}^{n-1} z^{n-1-k} f^{(k)}(0),
$$

with respect to $z$, whenever the integral on the left-hand side of (2.21) exists, it is obtained that

$$
\begin{aligned}
\frac{d^{m}}{(d z)^{m}} & \int_{0}^{+\infty} \frac{d^{n}}{(d \tau)^{n}} f(\tau) e^{-z(\tau-t)} d \tau \\
= & \int_{0}^{+\infty}(t-\tau)^{m} \frac{d^{n}}{(d \tau)^{n}} f(\tau) e^{-z(\tau-t)} d \tau \\
= & \frac{d^{m}}{(d z)^{m}}\left[z^{n} \tilde{f}(z) e^{z t}\right] \\
& -\sum_{k=0}^{m}\left(\begin{array}{c}
m \\
k
\end{array}\right) \frac{d^{m-k} e^{z t}}{(d z)^{m-k}} \sum_{r=k}^{n-1} \frac{r !}{(r-k) !} z^{r-k} f^{(n-1-r)}(0) .
\end{aligned}
$$

On the other hand, from the ordinary integral calculus, it is well known that if a function $t \mapsto \phi(t)$ is Riemann integrable over any finite time-interval $(0, t)$, the function $t \mapsto \int_{0}^{t} \phi(\tau) d \tau$ is continuous on, more precisely Riemann integrable over that time-interval $(0, t)$. Hence, for all Riemann integrable functions $t \mapsto \phi(t)$ over $(0, t)$, the $n$th order integral $\int_{0}^{t} \int_{0}^{t} \cdots \int_{0}^{t} \phi(t)(d t)^{n}$ is defined. The Laplace transform $\tilde{f}(z)$ of $f(t)=\int_{0}^{t} \int_{0}^{t} \cdots \int_{0}^{t} \phi(t)(d t)^{n}$, as it is well known, is $\tilde{f}(z)=z^{-n} \tilde{\phi}(z)$.

Accordingly, if the point $z=0$ is the $n$ th-order pole of a function $z \mapsto z^{-n} \tilde{\phi}(z) e^{z t}$, then in view of the fact that a function residue at the point $z=0$ is by definition

$$
\operatorname{Res}_{z=0}\left[z^{-n} \tilde{\phi}(z) e^{z t}\right]=\frac{1}{2 \pi i} \int_{G}^{\cup} z^{-n} \tilde{\phi}(z) e^{z t} d z,
$$

clearly under assumption that inside a domain bounded by $G$, as well as on its boundary, except for the singularity $z=0$, there is no other singularities of the function $z^{-n} \tilde{\phi}(z) e^{z t}$, that is,

$$
\operatorname{Res}_{z=0}\left[z^{-n} \tilde{\phi}(z) e^{z t}\right]=\frac{1}{(n-1) !} \lim _{z \rightarrow 0} \frac{d^{n-1}}{(d z)^{n-1}}\left\{z^{n}\left[z^{-n} \tilde{\phi}(z) e^{z t}\right]\right\},
$$

it follows from the result (2.22) that for $m=n-1$

$$
\frac{1}{2 \pi i} \int_{G}^{\cup} \frac{\tilde{\phi}(z)}{z^{n}} e^{z t} d z=\frac{1}{(n-1) !} \lim _{z \rightarrow 0} \int_{0}^{+\infty}(t-\tau)^{n-1} \phi(\tau) e^{-z(\tau-t)} d \tau,
$$


considering the fact that for every $k=0,1,2, \ldots, n-1$

$$
\begin{gathered}
\left.\lim _{z \rightarrow 0} \sum_{r=k}^{n-1} \frac{r !}{(r-k) !} z^{r-k}\left[\frac{d^{n-1-r}}{(d t)^{n-1-r}} \int_{0}^{t} \int_{0}^{t} \cdots \int_{0}^{t} \phi(t)(d t)^{n}\right]\right|_{t=0} \\
=\left.k !\left[\frac{d^{n-1-k}}{(d t)^{n-1-k}} \int_{0}^{t} \int_{0}^{t} \cdots \int_{0}^{t} \phi(t)(d t)^{n}\right]\right|_{t=0}=0 .
\end{gathered}
$$

In the case in which the function $z \mapsto \tilde{\phi}(z)$ is a regular one in the half-plane $\operatorname{Re} z>c$ $\left(c \in \mathbb{R}_{+}^{1}\right.$ ), it is obtained by application of the inversion theorem that

$$
\frac{1}{2 \pi i} \int_{c-i \infty}^{c+i \infty} \frac{\tilde{\phi}(z)}{z^{n}} e^{z t} d z=\frac{1}{(n-1) !} \int_{0}^{t}(t-\tau)^{n-1} \phi(\tau) d \tau .
$$

In addition, if all singularities of $\tilde{\phi}(z)$ are in the half-plane $\operatorname{Re} z<c^{\prime}\left(c^{\prime} \in \mathbb{R}_{-}^{1}\right)$ and $\lim _{z \rightarrow+\infty}\left[z^{-n} \tilde{\phi}(z)\right]=0$ for $\operatorname{Re} z<c$, then by the result of Theorem 1.2, it follows that

$$
\begin{array}{r}
\left|\frac{1}{(n-1) !} \lim _{z \rightarrow 0} \int_{0}^{+\infty}(t-\tau)^{n-1} \phi(\tau) e^{-z(\tau-t)} d \tau-\frac{1}{(n-1) !} \int_{0}^{t}(t-\tau)^{n-1} \phi(\tau) d \tau\right| \\
=\left|\frac{1}{2 \pi i} \int_{c^{\prime}-i \infty}^{c^{\prime}+i \infty} \frac{\tilde{\phi}(z)}{z^{n}} e^{z t} d z\right|=e^{c^{\prime} t}\left|\frac{1}{2 \pi i} \int_{c^{\prime}-i \infty}^{c^{\prime}+i \infty} \frac{\tilde{\phi}(z)}{z^{n}} e^{i(\operatorname{Im} z) t} d z\right| \leq a e^{c^{\prime} t},
\end{array}
$$

where $a=(1 / 2 \pi i) \int_{\mathcal{c}^{\prime}-i \infty}^{c^{\prime}+i \infty}\left|z^{-n}\right||\tilde{\phi}(z)| d z$, that is,

$$
\left|\frac{1}{(n-1) !} \int_{t}^{+\infty}(t-\tau)^{n-1} \phi(\tau) d \tau\right| \leq a e^{c^{\prime} t} .
$$

On the basis of the preceding results and an integral value of the function $\tau \mapsto$ $\tau^{n-1} e^{-\tau}[5]$

$$
\int_{0}^{+\infty} \tau^{n-1} e^{-\tau} d \tau=(n-1) !
$$

it follows that if $\tilde{\boldsymbol{\Phi}}_{s}(z)$, as the Laplace transform of the fundamental matrix $\boldsymbol{\Phi}_{s}(t)$ of system (2.1), is a regular-analytic function in the half-plane $\operatorname{Re} z>c\left(c \in \mathbb{R}_{+}^{1}\right)$, more precisely if all singularities of the function $\tilde{\boldsymbol{\Phi}}_{s}(z)$ are in the half-plane $\operatorname{Re} z<c^{\prime}$ (where $c^{\prime} \in \mathbb{R}_{-}^{1}$ ) and $\lim _{z \rightarrow+\infty}\left[z^{-n} \tilde{\boldsymbol{\Phi}}_{s}(z)\right]=0$ for $\operatorname{Re} z<c$, then it holds that

$$
\begin{aligned}
\int_{t}^{+\infty}(t-\tau)^{n-1} \boldsymbol{\Phi}_{S}(\tau) d \tau & =\frac{1}{2 \pi i} \int_{c^{\prime}-i \infty}^{c^{\prime}+i \infty} \frac{\tilde{\boldsymbol{\Phi}}_{s}(z)}{z^{n}} e^{i(\operatorname{Im} z) t} d z \int_{0}^{-\infty}\left(c^{\prime} \tau\right)^{n-1} e^{c^{\prime}(t-\tau)} d\left(c^{\prime} \tau\right) \\
& =\frac{\left(c^{\prime}\right)^{n}}{2 \pi i} \int_{c^{\prime}-i \infty}^{c^{\prime}+i \infty} \frac{\tilde{\boldsymbol{\Phi}}_{s}(z)}{z^{n}} e^{i(\operatorname{Im} z) t} d z \int_{t}^{+\infty}(t-\tau)^{n-1} e^{c^{\prime} \tau} d \tau,
\end{aligned}
$$

that is,

$$
\int_{t}^{+\infty}(t-\tau)^{n-1} \boldsymbol{\Phi}_{S}(\tau) d \tau=\mathbf{Y}(t) \int_{t}^{+\infty}(t-\tau)^{n-1} e^{c^{\prime} \tau} d \tau
$$


where $\mathbf{Y}(t)=\left(\left(c^{\prime}\right)^{n} / 2 \pi i\right) \int_{\mathcal{c}^{\prime}-i \infty}^{c^{\prime}+i \infty} z^{-n} \tilde{\boldsymbol{\Phi}}_{s}(z) e^{i(\operatorname{Im} z) t} d z$, more precisely,

$$
\lim _{t \rightarrow+\infty} \boldsymbol{\Phi}_{s}(t)=\mathbf{0}
$$

3. Some comments on impulsive behavior of linear singular systems. The preceding results are up to a point in collision with the one ad hock result of the theory of time-invariant linear singular control systems, and which in direct connection with socalled impulsive behavior of singular systems [3]. In [3], the result (taken over from [8]) was presented, which makes equal a number of impulsive modes, more precisely of infinite frequency modes, in a solution formulation for linear time-invariant singular systems without delay, to the algebraic expression value $\operatorname{rank} \mathbf{P}-\operatorname{degree}\{\operatorname{det}(z \mathbf{P}-\mathbf{A})\}$. For the illustration of the fact that such result is unfounded, the following example [7] is indicative.

EXAMPLE 3.1. Since the highest degree of the complex variable $z$ in the polynomial function $\pi(z)=\operatorname{det}(z \mathbf{P}-\mathbf{A})$ of a system of differential-algebraic equations

$$
\begin{gathered}
\frac{d}{d t} \mathbf{q}(t)=\mathbf{x}(t), \\
{\left[\begin{array}{llll}
1 & 0 & 0 & 0 \\
0 & 1 & 0 & 0 \\
0 & 0 & 1 & 0 \\
0 & 0 & 0 & 0
\end{array}\right] \mathbf{x}(t)-\left[\begin{array}{cccc}
a & b & c & 0 \\
e & f & g & h \\
i & j & k & 0 \\
m & 0 & n & 0
\end{array}\right] \mathbf{q}(t)=\mathbf{0},}
\end{gathered}
$$

for $n j \neq-m b$ and $h \neq 0$, is

$$
\text { degree }\{\pi(z)\}=\text { degree }\{-h\{m[(z-k) b+j c]+n[(z-a) j+b i]\}\}=1,
$$

and the rank of the singular matrix $\mathbf{P}$ of the system: $\operatorname{rank} \mathbf{P}=3<4$, on the one hand, it follows that

$$
\operatorname{rank} \mathbf{P} \text { - degree }\{\operatorname{det}(z \mathbf{P}-\mathbf{A})\}=3-1=2 .
$$

On the other hand, in an explicitly obtained solution formulation for the given system of differential-algebraic equations

$$
\begin{gathered}
q_{1}(t)=q_{1}\left(t_{0}\right) e^{\alpha\left(t-t_{0}\right)} ; \\
q_{2}(t)=\frac{m(k-a)+m^{2} c / n-n i}{n j+m b} q_{1}(t) ; \\
q_{3}(t)=-\frac{m}{n} q_{1}(t) ; \\
q_{4}(t)=\frac{1}{h}\left[(\alpha-f) \frac{m(k-a)+m^{2} c / n-n i}{n j+m b}+\left(\frac{g m}{n}-e\right)\right] q_{1}(t),
\end{gathered}
$$

where $\alpha=(n(j a-b i)+m(b k-j c) /(n j+m b))$, there are no infinite frequency modes (impulsive modes) but they are present only finite frequency modes (exponential modes).

An idea on the presence of infinite frequency modes (impulsive modes) in a solution formulation for linear time-invariant singular systems of differential equations 
without delay is among other things a consequence of an insufficiently exact application of the Laplace transform in the course of solving a singular system of differential equations $\mathbf{N}_{2}(t)=\mathbf{x}_{2}(t)+\mathbf{B u}(t)$, where $n \times n$ matrix $\mathbf{N}$ is a nilpotent matrix with index of nilpotency $v$, and $\mathbf{B}$ is an arbitrary rectangular $m \times n$ matrix, as a subsystem of the standard (Weirestrass) canonical form of linear time-invariant singular systems. Namely, the solution of the aforementioned system of differential equations is $\mathbf{X}_{2}(t)=-\sum_{i=0}^{v-1} \mathbf{N}^{i} \mathbf{B} \mathbf{u}^{(i)}(t)$, since

$$
\begin{aligned}
\mathbf{N} \dot{\mathbf{x}}_{2}(t) & =-\sum_{i=0}^{v-1} \mathbf{N}^{i+1} \mathbf{B} \mathbf{u}^{(i+1)}(t)=-\sum_{i=1}^{v} \mathbf{N}^{i} \mathbf{B} \mathbf{u}^{(i)}(t) \\
& =-\sum_{i=0}^{v-1} \mathbf{N}^{i} \mathbf{B} \mathbf{u}^{(i)}(t)+\mathbf{B u}(t)=\mathbf{x}_{2}(t)+\mathbf{B u}(t) .
\end{aligned}
$$

However, in [3] (taken over from [2]) the following result was presented:

$$
\mathbf{x}_{2}(t)=-\sum_{i=0}^{v-1} \delta^{(i)}(t) \mathbf{N}^{i+1} \mathbf{x}_{2}(0)-\sum_{i=0}^{v-1} \mathbf{N}^{i} \mathbf{B u}^{(i)}(t),
$$

where $\delta^{(i)}(t)$ is $i$ th derivative of Dirac's delta distribution, in which there exist infinite frequency modes (impulsive modes). From what follows it will be seen that even if the inclusion of distributions in a solution formulation for linear time-invariant singular systems would be acceptable, this result must be corrected so that the system solution, under the aforementioned circumstance, is

$$
\mathbf{x}_{2}(t)=-\sum_{i=0}^{v-1} \delta^{(i)}(t) \mathbf{N}^{i+1}\left[\mathbf{x}_{2}(0)+\sum_{i=0}^{v-1} \mathbf{N}^{i} \mathbf{B} \mathbf{u}^{(i)}(0)\right]-\sum_{i=0}^{v-1} \mathbf{N}^{i} \mathbf{B} \mathbf{u}^{(i)}(t),
$$

that is,

$$
\mathbf{x}_{2}(t)=\sum_{i=0}^{v-1} \mathbf{N}^{i}\left\{\mathbf{N}[1-\chi(t)]^{(i+1)}\left[\mathbf{x}_{2}(0)+\sum_{j=0}^{v-i-1} \mathbf{N}^{j} \mathbf{B} \mathbf{u}^{(j)}(0)\right]-\mathbf{B} \mathbf{u}^{(i)}(t)\right\},
$$

where $\chi(t)$ is the unit step function, since

$$
\sum_{i=0}^{v-1} \delta^{(i)}(t) \mathbf{N}^{i+1} \sum_{i=0}^{v-1} \mathbf{N}^{i} \mathbf{B} \mathbf{u}^{(i)}(0)=\sum_{i=0}^{v-1} \mathbf{N}^{i+1} \delta^{(i)}(t) \sum_{j=0}^{v-i-1} \mathbf{N}^{j} \mathbf{B} \mathbf{u}^{(j)}(0) .
$$

By application of the right-hand Laplace transform to the pure singular system $\mathbf{N}(d / d t) \mathbf{X}_{2}(t)=\mathbf{x}_{2}(t)+\mathbf{B u}(t)$, for example, it is obtained that

$$
\begin{aligned}
\mathbf{X}_{2}(z) & =(z \mathbf{N}-\mathbf{E})^{-1}\left[\mathbf{N x}_{2}(0)+\mathbf{B u}(s)\right] \\
& =-\sum_{i=0}^{v-1} \mathbf{N}^{i} z^{i}\left[\mathbf{N x}_{2}(0)+\mathbf{B u}(s)\right],
\end{aligned}
$$


that is,

$$
\begin{aligned}
\tilde{\mathbf{x}}_{2}(z)= & \sum_{i=0}^{v-1} \mathbf{N}^{i} z^{i}\left[\mathbf{N} \sum_{i=0}^{v-1} \mathbf{N}^{i} \mathbf{B} \mathbf{u}^{(i)}(0)-\mathbf{B} \tilde{\mathbf{u}}(z)\right] \\
& -\sum_{i=0}^{v-1} \mathbf{N}^{i+1} z^{i}\left[\mathbf{x}_{2}(0)+\sum_{i=0}^{v-1} \mathbf{N}^{i} \mathbf{B} \mathbf{u}^{(i)}(0)\right] \\
= & -\mathbf{B} \tilde{\mathbf{u}}(z)-\sum_{i=1}^{v-1} \mathbf{N}^{i} \mathbf{B}\left[z^{i} \tilde{\mathbf{u}}(z)-\sum_{j=0}^{i-1} z^{j} \mathbf{u}^{(i-1-j)}(0)\right] \\
& -\sum_{i=0}^{v-1} \mathbf{N}^{i+1} z^{i}\left[\mathbf{x}_{2}(0)+\sum_{i=0}^{v-1} \mathbf{N}^{i} \mathbf{B} \mathbf{u}^{(i)}(0)\right] .
\end{aligned}
$$

Finally, by use of the inversion Laplace theorem, clearly under the condition that

$$
\mathbf{x}_{2}(0)=-\sum_{i=0}^{v-1} \mathbf{N}^{i} \mathbf{B} \mathbf{u}^{(i)}(0),
$$

we obtain

$$
\mathbf{x}_{2}(t)=-\mathbf{B u}(t)-\sum_{i=1}^{v-1} \mathbf{N}^{i} \mathbf{B} \mathbf{u}^{(i)}(t)=-\sum_{i=0}^{v-1} \mathbf{N}^{i} \mathbf{B} \mathbf{u}^{(i)}(t),
$$

which is equivalent to explicitly obtained solution without infinite frequency modes (impulsive modes) on the one hand, and on the other satisfies the aforementioned condition.

4. Conclusion. It is an unquestionable fact that a class of singular systems of differential equations is more general with respect to a class of regular systems of differential equations. Accordingly, it is a question whether we can, in spite of all peculiarities of these two class of systems, apply the same methodology in the qualitative analysis of their dynamic behavior?

The results obtained in this paper give a solid base for formulating the answer to the former asked question. In other words, on the basis of our results, we can say that peculiarities of a class of linear time-invariant time-delayed singular systems are not of that generality which would require the new methodology in the analysis of dynamic behavior of that class of systems in comparison with that applied in the analysis of dynamic behavior of a class of linear time-invariant time-delayed regular systems.

\section{REFERENCES}

[1] R. Belman and K. L. Kuk, Differential-Difference Equations, The World, Moscow, 1967.

[2] L. Dai, Singular Control Systems, Lecture Notes in Control and Information Sciences, vol. 118, Springer-Verlag, Berlin, 1989.

[3] D. L. J. Debeljkovic, S. A. Milinkovic, and M. B. Jovanovic, Continuous-Time Linear Singular Systems, GIP-Culture, Belgrade, 1996.

[4] D. S. Mitrinović and J. D. Kečkić, Cauchyjev račun ostataka sa primenama [Cauchy's Calculus of Residues with Applications], Mathematical Problems and Expositions, vol. 8, Naučna Knjiga, Belgrade, 1978 (Serbian). 
[5] D. S. Mitrinović, D. Đ. Tošić, and R. R. Janić, Specijalne funkcije [Special Functions], 3rd ed., New Collection of Mathematical Problems, vol. 1, Naučna Knjiga, Belgrade, 1986 (Serbian).

[6] V. Rezvan, Absolyutnaya ustoichivost avtomaticheskikh sistem s zapazdyvaniem [The Absolute Stability of Automatic Systems with Delay], Nauka, Moscow, 1983 (Russian).

[7] B. V. Saric, Concept of energy hyper-stability of time-varying non-linear continuous-time control systems, Master's thesis, Faculty of Mechanical Engineering, University of Belgrade, Belgrade, 1999.

[8] G. C. Verghese, B. C. Lévy, and T. Kailath, A generalized state-space for singular systems, IEEE Trans. Automat. Control 26 (1981), no. 4, 811-831.

Branko SARic: Kirilo SAVic InStitute, 11000 Belgrade, V. STEPe 51, Serbia

E-mail address: bsaric@ptt.yu 


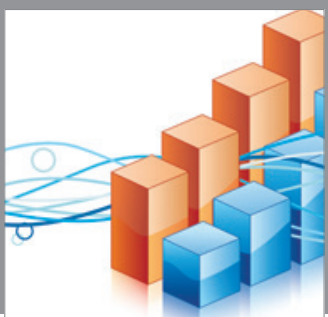

Advances in

Operations Research

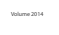

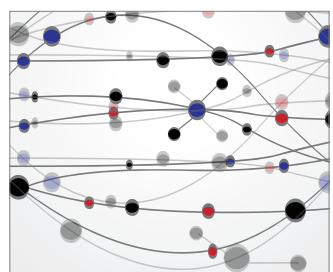

\section{The Scientific} World Journal
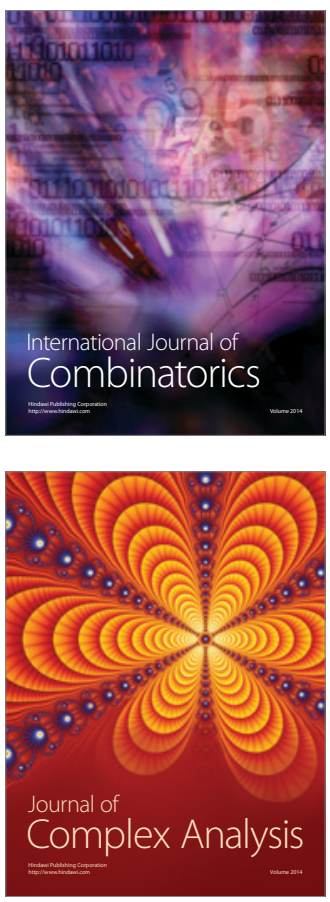

International Journal of

Mathematics and

Mathematical

Sciences
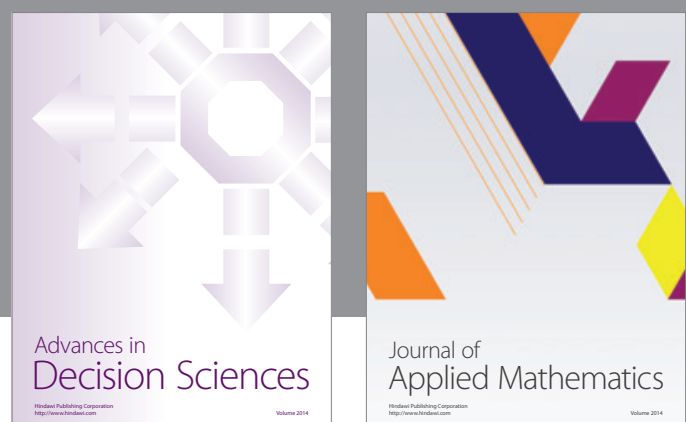

Journal of

Applied Mathematics
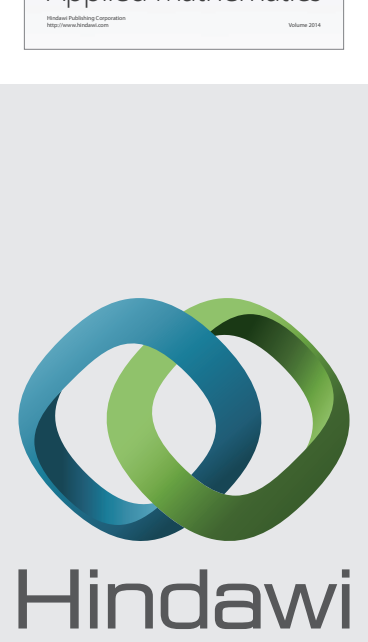

Submit your manuscripts at http://www.hindawi.com
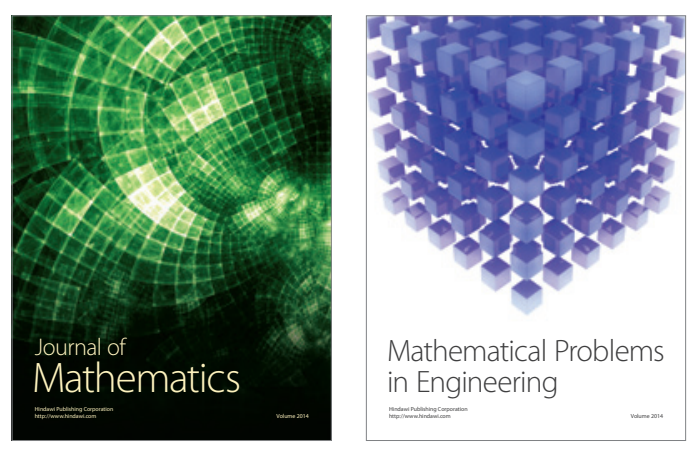

Mathematical Problems in Engineering
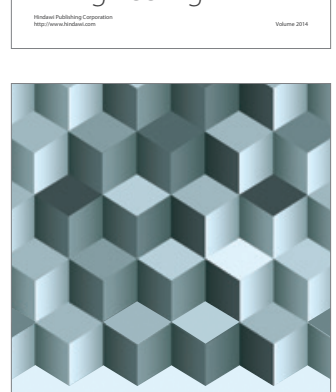

Journal of

Function Spaces
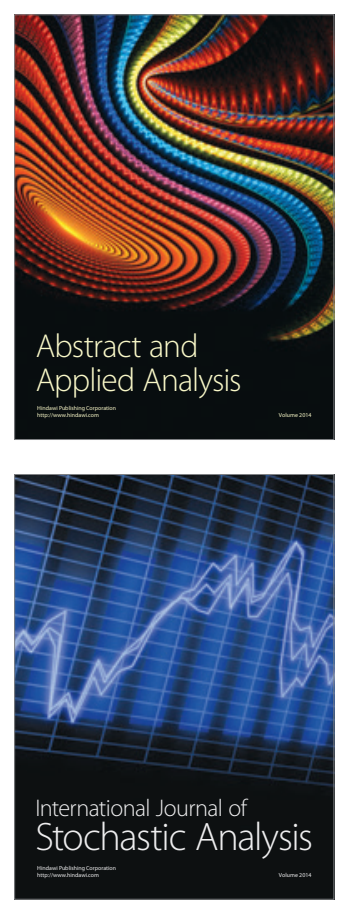

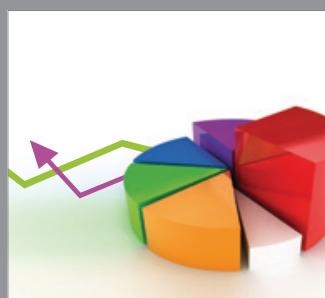

ournal of

Probability and Statistics

Promensencen
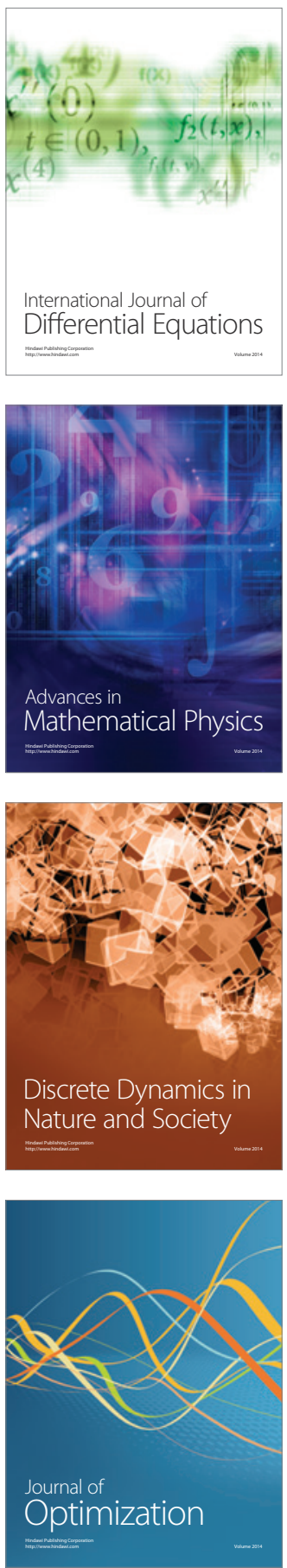